\title{
Phylogenetic analysis of Bunyamwera and Ngari viruses (family Bunyaviridae, genus Orthobunyavirus) isolated in Kenya
}

\author{
C. Odhiambo ${ }^{1,2,3 *}$, M. Venter ${ }^{2}$, O.Lwande ${ }^{1}$, R.Swanepoel ${ }^{2}$ and R. Sang ${ }^{1,3,4}$
}

\author{
${ }^{1}$ Human Health Division, International Centre of Insect Physiology and Ecology, Nairobi, Kenya \\ ${ }^{2}$ Zoonoses Research Unit, Department of Medical Virology, University of Pretoria, Pretoria, South Africa \\ ${ }^{3}$ Centre for Virus Research, Kenya Medical Research Institute, Nairobi, Kenya \\ ${ }^{4}$ Division of Emerging Infectious Disease, United States Army Medical Research Unit, Kenya
}

\begin{abstract}
Summary
Orthobunyaviruses, tri-segmented, negative-sense RNA viruses, have long been associated with mild to severe human disease in Africa, but not haemorrhagic fever. However, during a Rift Valley fever outbreak in East Africa in 1997-1998, Ngari virus was isolated from two patients and antibody detected in several others with haemorrhagic fever. The isolates were used to identify Ngari virus as a natural Orthobunyavirus reassortant. Despite their potential to reassort and cause severe human disease, characterization of orthobunyaviruses is hampered by paucity of genetic sequences. Our objective was to obtain complete gene sequences of two Bunyamwera virus and three Ngari virus isolates from recent surveys in Kenya and to determine their phylogenetic positioning within the Bunyamwera serogroup. Newly sequenced Kenyan Bunyamwera virus isolates clustered closest to a Bunyamwera virus isolate from the same locality and a Central African Republic isolate indicating that similar strains may be circulating regionally. Recent Kenyan Ngari isolates were closest to the Ngari isolates associated with the 1997-1998 haemorrhagic fever outbreak. We observed a temporal/geographical relationship among Ngari isolates in all three gene segments suggesting a geographical/temporal association with genetic diversity. These sequences in addition to earlier sequences can be used for future analyses of this neglected but potentially deadly group of viruses.
\end{abstract}

Key words: Arboviruses, bunyaviruses, viral haemorrhagic fever, virus infection, zoonoses.

\section{Introduction}

The Bunyaviridae family is divided into five genera; Orthobunyavirus, Phlebovirus, Nairovirus, Hantavirus and Tospovirus [1]. The largest genus, Orthobunyavirus, is composed of over 150 viruses that infect humans and

\footnotetext{
* Author for correspondence: Dr C. Odhiambo, Human Health Division, International Centre of Insect Physiology and Ecology, PO Box 1578, Kisumu 40100, Kenya .

(Email: coodhiambo@kemricdc.org)
}

are transmitted by mosquitoes, midges and ticks from reservoir animals like rodents and livestock. Members of Orthobunyavirus are tri-segmented, negative-sense RNA viruses responsible for mild to severe human and animal diseases. The $\mathrm{L}$ (large) segment encodes a large protein that consists of the RNA-dependent RNA polymerase activity for replication and transcription of genomic RNA segments. The M (medium) segment encodes a precursor polypeptide which yields the virion surface glycoproteins $\mathrm{Gn}$ and $\mathrm{Gc}$ and a nonstructural protein NSm, and the S (small) segment 
encodes the nucleocapsid (NC) and a non-structural protein (NSs) in overlapping reading frames [2]. Bunyamwera virus is the prototype virus of the Orthobunyavirus genus as well as the Bunyaviridae family of arboviruses. Bunyamwera virus is associated with febrile illness, headache, arthralgia, rash and infrequent central nervous system involvement [3]. Viruses of the Orthobunyavirus genus were generally not associated with haemorrhagic symptoms until Ngari virus was implicated in haemorrhagic fever outbreaks in Kenya and Somalia, and retrospectively in Sudan [4-6]. Ngari virus was determined to be a reassortment between two segmented viruses (Bunyamwera and Batai) cocirculating within the same environment. Subsequent analysis of isolated sequences from outbreak samples showed that the $\mathrm{L}$ and $\mathrm{S}$ segment sequences closely matched those of Bunyamwera virus while the $\mathrm{M}$ segment was identical to that of Batai virus [5].

Surveillance activities during Rift Valley fever outbreaks have demonstrated co-circulation of arboviruses including Bunyamwera virus [7]. Additionally, ongoing inter-epidemic surveillance activities have indicated continued intense transmission of Bunyamwera virus [8]. Co-circulation of viruses within the same serogroup is likely to provide opportunities for genetic reassortments. Efforts to bring these infections under control and predict their emergence will not be successful unless there is a full understanding of how these viruses are maintained and transmitted within the environment. However, characterization of these emergent arboviral species has been hampered by paucity of genetic sequences making it impossible to accurately estimate their evolutionary trend and public health burden. We have recently isolated Bunyamwera and Ngari viruses from surveillance exercises in Kenya and identified them based on short diagnostic sequences $[8,9]$. Our main objective was to provide complete coding sequence of some of these isolates as well as to investigate their phylogenetic positioning within the Bunyamwera serogroup, based on partial and complete genome sequences of similar virus strains and other selected orthobunyaviruses.

\section{Methods}

Bunyamwera and Ngari virus isolates (Table 1) obtained from previous surveillance exercises in northern Kenya were inoculated into flasks containing confluent Vero cells and observed daily for cytopathic effects (CPE). Viruses were harvested when more than $75 \%$ of cells showed CPE. The supernatants were aliquoted into cryotubes and stored at $-70{ }^{\circ} \mathrm{C}$, until use.
For RNA extraction, the MagNA Pure LC RNA isolation kit I (Roche Applied Science, USA) was used. cDNA was synthesized using the Transcriptor first-strand cDNA synthesis kit (Roche Applied Science) with random hexamers followed by PCR using Phusion high-fidelity PCR kit (Finnzyme Ltd, Finland) and appropriate primers. Overlapping primers (Supplementary Table S1) for each segment were either designed based on sequences of Bunyamwera, Batai and Ngari viruses available in GenBank or obtained from previous publications $[10,11]$. Amplified DNA fragments were visualized by electrophoresis on a 1.5\% agarose gel. Amplified DNA fragments were purified and prepared for sequencing using ExoSAP-IT PCR clean-up kit (USB Corp., USA) according to manufacturer's instructions and stored at $-20^{\circ} \mathrm{C}$.

Sequencing was performed using sets of overlapping primers for the $\mathrm{S}, \mathrm{M}$ and $\mathrm{L}$ segments as designed previously (Supplementary Table S1) using the Big Dye v. 3.1 kit (Applied Biosystems, USA) and run on the 3500XL Genetic Analyzer (Applied Biosystems). The sequences obtained were cleaned and edited using Bioedit software (USA) for both reads from the forward and reverse primers. Sequences obtained were subjected to Basic Local Alignment Search Tool (BLAST) searches in NCBI GenBank (http://www.ncbi.nlm.nih. gov/blast/Blast) to identify similar sequences. The clean sequences of each segment were aligned against a selection of corresponding segment sequences of Bunyamwera serogroup viruses, including Bunyamwera, Batai and Ngari viruses, using the multiple alignment fast Fourier transform (MAFFT) sequence alignment program [12]. Phylogenetic trees were constructed using maximum likelihood algorithm and analysed with 1000 replicates for bootstrap testing in Molecular Evolutionary Genetics Analysis (MEGA) v. 5.20 software [13]. La Crosse virus of the California serogroup was used as the out-group for phylogenetic analyses of all three segments of Bunyamwera and Ngari viruses. Nucleotide and amino acid similarities and genetic distances between the Kenyan isolates and selected isolates from diverse regions were computed in MEGA v. 5.20 using the p-distance method [13].

\section{Results and Discussion}

The complete $\mathrm{S}, \mathrm{M}$ and $\mathrm{L}$ segment coding regions (704, 4302 and $6717 \mathrm{nt}$, respectively) were sequenced for all five isolates. For this analysis, we excluded the $3^{\prime}$ and $5^{\prime}$ non-coding regions. The gene sequences 
Table 1. Bunyamwera and Ngari virus isolates from Kenya sequenced in the study

\begin{tabular}{llllll}
\hline \hline Virus & Code & Strain & Site of isolation & Year & Isolation source \\
\hline Bunyamwera & BUNV_11232 & BUNV_GSA/S4/11232_WT & Garissa & 2009 & Aedes mcintoshi \\
& BUNV_12060 & BUNV_MGD/S1/12060_WT & Magadi & 2009 & Anopheles funestus \\
Ngari & NRIV_19801 & NRIV_TND/S1/19801_WT & Tana-delta & 2011 & Anopheles funestus \\
& NRIV_5170 & NRIV_GSA/TS7/5170_WT & Garissa & 2009 & Amblyomma gemma \\
& NRIV_5242 & NRIV_ISL/TS2/5242_WT & Isiolo & 2009 & Rhipicephalus pulchellus \\
\hline \hline
\end{tabular}

determined in this study are deposited in GenBank under the following accession numbers ( $\mathrm{S}$ segment, M segment, L segment):

\section{KM507344, KM507340 and KM507338 (BUNV_ MGD_S1_12060_WT); \\ KM507345, KM507339 and KM507337 (BUNV_ GSA_S4_11232_WT); \\ KM507343, KM514679 and KM507335 (NRIV_ TND_S1_19801_WT); \\ KM507341, KM514677 and KM507336 (NRIV_ GSA_TS7_5170_WT); \\ KM507342, KM514678 and KM507334 (NRIV_ ISL_TS2_5242_WT).}

Phylogenetic analyses reveal that within all three genomic segments, Bunyamwera virus isolates sequenced in the current study clustered together with an isolate in GenBank from the same locality (BUNV AMH001130) with strong bootstrap support. The newly sequenced Kenyan Bunyamwera virus isolates possess high nucleotide sequence identities of over $99 \cdot 0 \%(\mathrm{~S}), 99 \cdot 4 \%(\mathrm{M})$ and $99 \cdot 4 \%(\mathrm{~L})$ compared to the previous Kenyan isolate (BUNV_AMH001130). Similarly, the newly sequenced Kenyan Bunyamwera virus isolates possess high percentage amino acid sequence identities of over $99 \cdot 5 \%$ (N ORF), $99 \cdot 0 \%$ (NSs ORF), $99 \cdot 5 \%$ (M polyprotein ORF) and $98 \cdot 8 \%$ (L protein ORF) compared to the previous Kenyan isolate. These results indicate that similar Bunyamwera virus strains may be circulating within Kenya and also regionally considering the close association with the 1994 Bunyamwera virus isolate from the Central African Republic (BUNV_ArB29051).

Similarly for Ngari virus, the newly sequenced Kenyan isolates, regardless of the genomic segment, clustered with other Ngari virus isolates (Fig. 1a-c). The newly sequenced Kenyan Ngari virus isolates were closer to Ngari virus isolates associated with the 1997-1998 haemorrhagic fever outbreak in East Africa (NRIV_9800535 and NRIV_9800521) with strong bootstrap support particularly in analyses of the $\mathrm{M}$ and $\mathrm{L}$ segments (Fig. 1b,c). This observation suggests that the current Kenyan Ngari virus isolates may have been introduced into Kenya from the Kenya-Somali border. The newly sequenced Kenyan Ngari virus isolates also possess high nucleotide sequence identities of over $98.5 \%(\mathrm{~S}), 97.0 \%(\mathrm{M})$ and 97.3\% (L) compared to other Ngari virus strains regardless of year and place of isolation. Similarly, the newly sequenced Kenyan Ngari virus strains possess high amino acid sequence identities of over $99 \cdot 1 \%$ (N ORF), 100\% (NSs ORF), 98.4\% (M polyprotein $\mathrm{ORF}$ ) and $99 \cdot 1 \%$ (L protein) compared to other Ngari virus isolates. The complete sequence data for the three additional isolates of Ngari virus support previous findings that this virus is a reassortant [4-6].

Of additional interest, we observed temporal/ geographical clustering of Ngari isolates in all three phylogenetic trees showing that isolates from the same region or outbreak year generally group together within their own strongly supported independent groupings (Fig. 1 $a-c$ ). Our findings suggest a geographical/temporal association with genetic diversity between strains of Ngari virus. Similar observations have been made for Batai virus in which correlation between geographical and genetic diversity has been suggested [10, 14]. Batai virus isolates from Europe, Asia and Africa generally cluster independently within strongly supported groups with country-specific viruses clustering closest [14]. However, a more in-depth analysis including identification of signature motifs that might be representative of a given geographical/temporal clade of these viruses is restricted by paucity of genetic sequences in GenBank. Furthermore, there is need for more studies on pathogenesis and distribution of these viruses. While public health efforts have focused on wellcharacterized viruses such as Rift Valley Fever, West Nile, chikungunya and dengue viruses, the emergence of orthobunyaviruses such as Ngari and Schmallenberg viruses as human and veterinary pathogens, respectively, emphasize the need for in-depth characterization and determination of their true public health impact. 
(a)

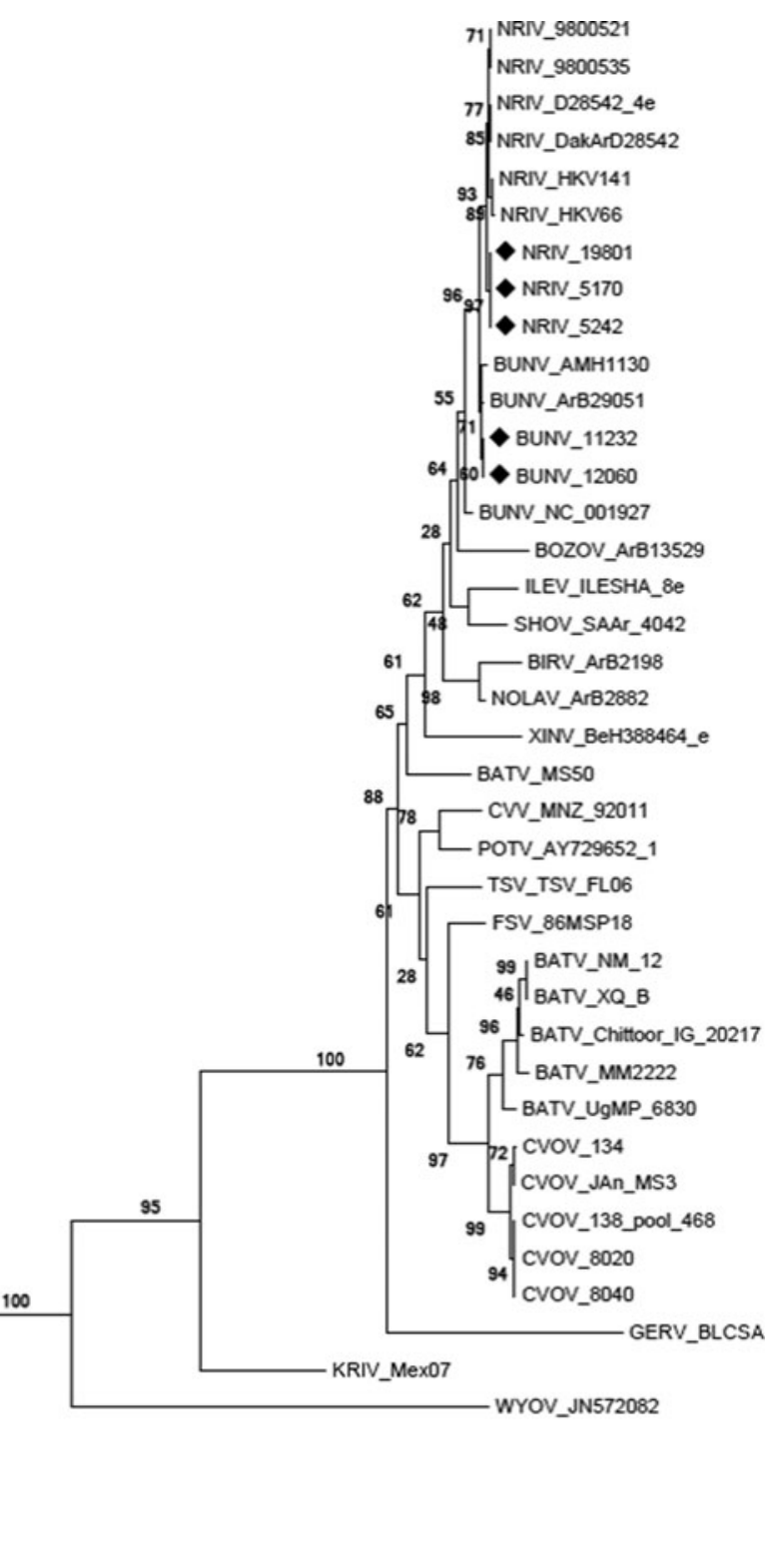

Fig. 1. Phylogenies of the S, M and L segments of the genus Orthobunyavirus. According to a limited diversity of data that are available in GenBank, the maximum likelihood method was used to derive phylogenies from $(a)$ the N gene nucleotide ORF (S segment, $702 \mathrm{nt}$ ), (b) M polyprotein ORF (M segment, $4302 \mathrm{nt}$ ) and (c) L polymerase protein ORF (L segment, $6717 \mathrm{nt})$ and ORFs of selected orthobunyaviruses. Phylogenies were rooted using the La Crosse virus sequence. Bootstrap values that were determined by 1000 replicates are shown. Scale bars represent the number of nucleotide substitutions per site. Sequences determined as a part of this study are indicated by a rectangle. All Orthobunyavirus abbreviations are used according to the International Committee on Taxonomy of Viruses (ICTV, 2005). BATV, Batai virus; BIRV, Birao virus; BOZOV, Bozo virus; BUNV, Bunyamwera virus; CVV, Cache Valley virus; CVOV, Calovo virus; FSV, Fort Sherman virus; GERV, Germiston virus; ILEV, Ilesha virus; KIRV, Kairi virus; LACV, La Crosse virus; NDOV, Nyando virus; NOLAV, Nola virus; NRIV, Ngari virus; PGAV, Pongola virus; POTV, Potosi virus; SHOV, Shokwe virus; TSV, Tensaw virus; WYOV, Wyomyia virus; XINV, Xingu virus. S segment accession numbers: BATV_Chittoor_IG_20217 (JX846598), BATV_MM2222 (JX846595), BATV_MS50 (JX846604), BATV_NM_12 (KJ187040), BATV_UgMP_6830 (JX846601), BATV_XQ_B (KJ398936), BIRV_ArB2198 (AM711131), BOZOV_ArB13529 (AM711132), BUNV_ArB29051 (AM709778), BUNV_NC001927, BUNV_AMH1130 (JF961342), CVOV_134 (KJ542624), CVOV_138_pool_468 (KC608157), CVOV_8020 (KJ542630), CVOV_8040 (KJ542633), CVOV_JAn_MS3 (KJ542627), CVV_MNZ_92011 (KC436108), FSV_86MSP18 (EU564829), GERV_BLCSA (M19420), ILEV_ILESHA_8e (KC608151), KRIV_Mex07 (EU879063), LACV_Dallas_TX_2009 (GU591164), NOLAV_ArB2882 (AM711134), NRIV_9800521 (JX857325), NRIV_9800535 (JX857328), NRIV_D28542_4e (KC608154), NRIV_DakArD28542 (JX857316), NRIV_HKV141 (JX857322), NRIV_HKV66 (JX857319), NRIV_Adrar (KJ716848), PGAV_SAAr1 (EU564828), POTV_AY729652_1 (AY729652), SHOV_SAAr_4042 (EU564831), TSV_TSV_FL06 (FJ943507), XINV_BeH388464_e (EU564830). 
(b)

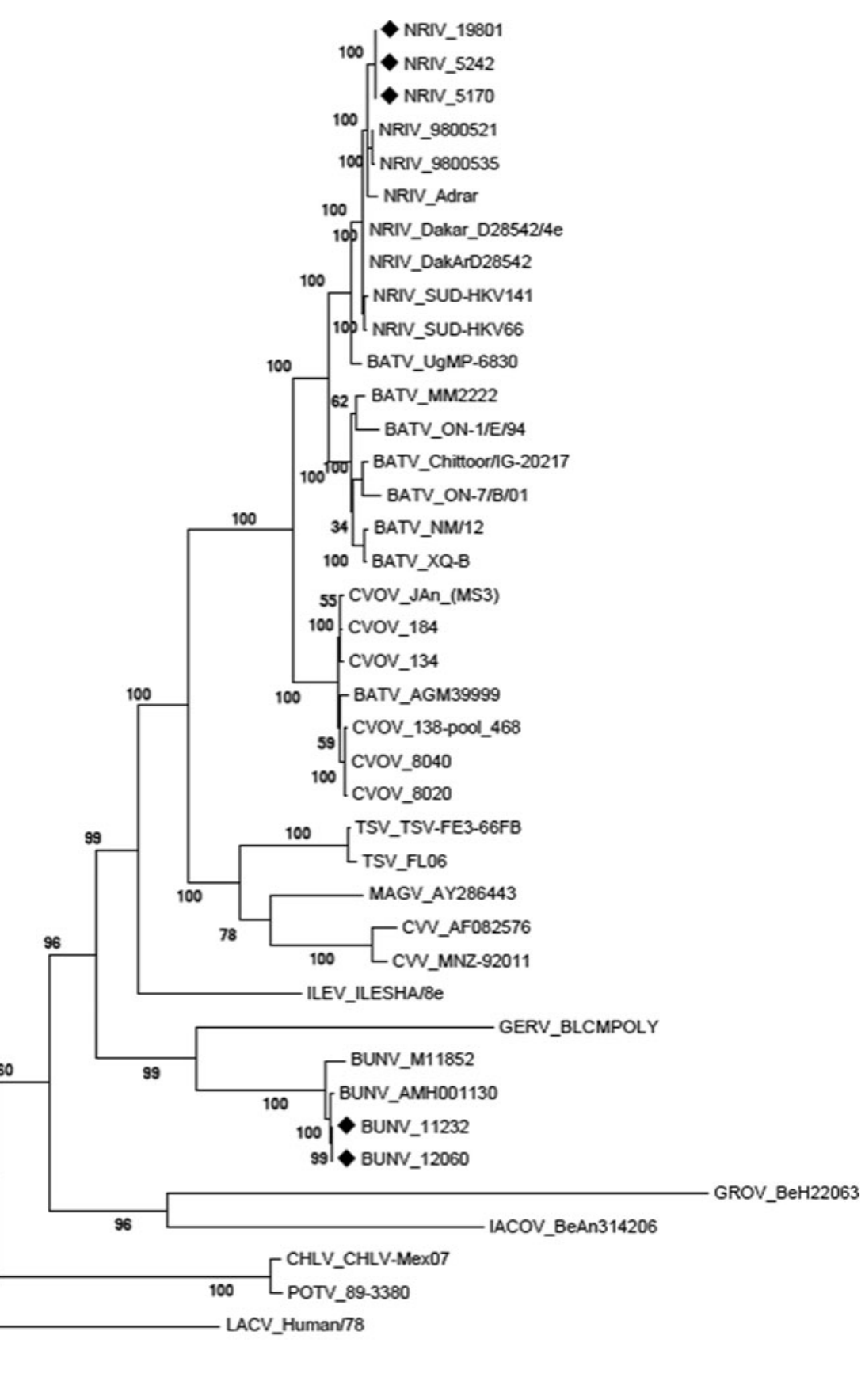

Fig. 1b. $M$ segment accession numbers: BATV_ON_7_B_01 (AB257765), BATV_ON_1_E_94 (AB257764), BATV_NM_12 (KJ187039), BATV_XQ+B (KJ398937), BATV_UgMP_6830 (JX846602), BATV_Chittoor_IG_20217 (JX846599), BATV_MM2222 (JX846596), BATV_Italy_2009 (KC168047), BUNV_M11852 (M11852), BUNV_AMH001130 (JF961341), CHLV_CHLV_Mex07 (JN808310), CVV_MNZ_92011 (KC436107), CVV_AF082576 (AF082576), CVV_807270 (AF186243), CVOV_138_pool_468 (KC608156), CVOV_JAnMS3 (KJ542628),CVOV_134 (KJ542625), CVOV_184 (DQ334335), GROV_BeH22063 (AY380581), GERV_BLCMPOLY (M21951), IACOV_BeAn314206 (JN572066), ILEV_ILESHA_8e (KC608150), LACV_Human_78 (AF528166), MAGV_AY286443 (AY286443), NRIV_Dakar_D28542_4e (KC608153), NRIV_9800521 (JX857326), NRIV_9800535 (AY593725), NRIV_DakArD28542 (JX857317), NRIV_HKV141 (JX857323)， NRIV_HKV66 (JX857320), POTV_89_3380 (EU004189), TSV_FE3_66FB (FJ943508), TSV_FL06 (FJ943506), NRIV_Adrar (KJ716849). 
(c)

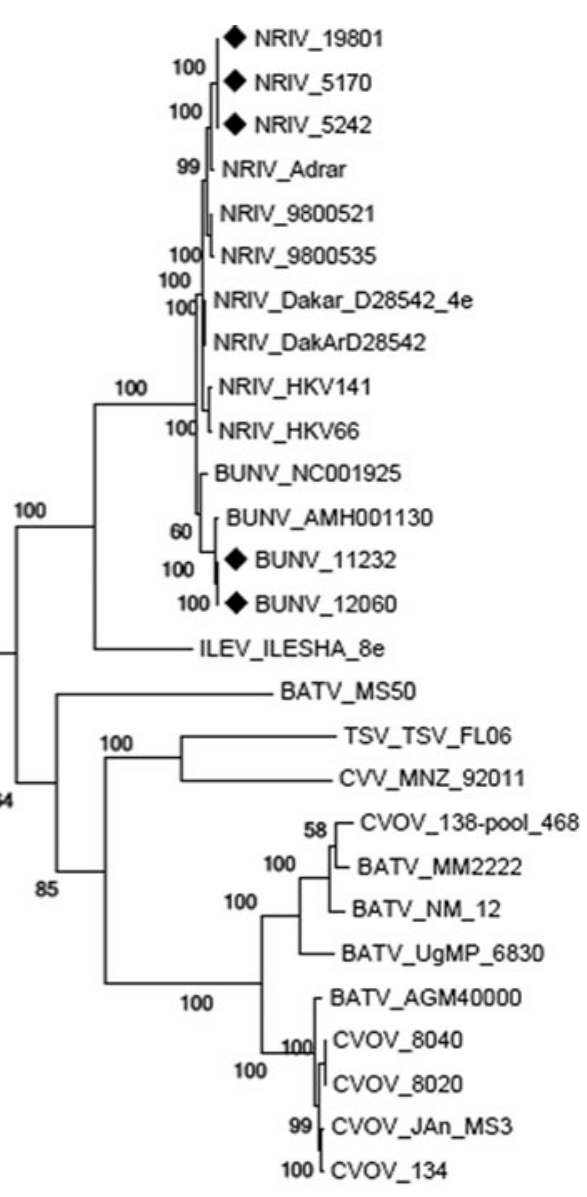

LACV_Human_78

0.2

Fig. 1c. L segment accession numbers: BATV_NM_12 (KJ187038), BATV_UgMP_6830 (JX846603), CVOV_138-pool_468 (JX846600), BATV_MM2222 (JX846597), BATV_Italy_2009 (KC168048), BUNV_AMH001130 (F961340), BATV_MS50 (JX846606)，BUNV_NC001925 (NC001925)，CVOV_8040 (KJ542635)，CVOV_8020 (KJ542632)，CVOV_JAn_MS3 (KJ542629), CVOV_134 (KJ542626), TSV_FL06 (FJ943509), CVV_MNZ_92011 (KC436106), ILEV_ILESHA_8e (KC608149), ILEV_R5964 (KF234075), LACV_Human_78 (AF528165), NRIV_D28542_4e (KC608152), NRIV_9800521 (JX857327), NRIV_9800535 (JX857330), NRIV_DakArD28542 (JX857318), NRIV_HKV141 (JX857324), NRIV_HKV66 (JX857321), NRIV_Adrar (KJ716850).

Additionally, as more sequences are continually generated, a more extensive survey with regard to sample size and geography is necessary to better understand the distribution of Ngari and Bunyamwera viruses.

\section{Acknowlegdements}

We acknowledge the technical assistance provided by Caroline Tigoi, David Tchouassi, Martin Mbaya and Felix Odhiambo all from the Martin Luscher Emerging Infectious Diseases Laboratory of the International Centre of Insect Physiology and Ecology. We also ac- knowledge the logistic support of Lillian Igweta, Lisa Omondi and Margaret Ochanda all of Capacity Building, ICIPE. The support through a scholarship to Collins Odhiambo by the Swedish International Development Cooperation Agency (SIDA) through the African Regional Postgraduate Programme in Insect Science (ARPPIS) is greatly acknowledged. The funders had no role in study design, data collection and analysis, decision to publish, or preparation of the manuscript. This paper is published with the permission of the Director of the Kenya Medical Research Institute. 


\section{Declaration of interest}

None.

\section{References}

1. Calisher CH. History, classification, taxonomy of viruses in the family Bunyaviridae. In: The Bunyaviridae, the Viruses. New York: Springer, 1996, pp. 1-17.

2. Soldan SS, Gonzalez-Scarano F. Emerging infectious diseases: the Bunyaviridae. Journal of NeuroVirology, 2005; 11: 412-423.

3. Gonzalez JP, Georges AJ. Bunyaviral fevers: Bunyamwera, Ilesha, Germiston, Bwamba and Tataguine. In: Monath TP, ed. The Arboviruses: Epidemiology and Ecology. Boca Raton, Florida: CRC Press, 1988, pp. 87-98.

4. Bowen MD, et al. A reassortant bunyavirus isolated from acute hemorrhagic fever cases in Kenya and Somalia. Virology 2001; 291: 185-190.

5. Briese T, et al. Batai and Ngari viruses: M segment reassortment and association with severe febrile disease outbreaks in East Africa. Journal of Virology 2006; 80: 5627-5630.

6. Gerrard S, et al. Ngari virus is a Bunyamwera virus reassortant that can be associated with large outbreaks of hemorrhagic fever in Africa. Journal of Virology 2004; 78: 8922-8926.
7. Crabtree M, et al. Arbovirus surveillance of mosquitoes collected at sites of active Rift Valley Fever virus transmission: Kenya, 2006-2007. Journal of Medical Entomology 2009; 46: 961-964.

8. Lutomiah $\mathbf{J}$, et al. Ticks and tick-borne viruses from livestock hosts in arid and semiarid regions of the eastern and northeastern parts of Kenya. Journal of Medical Entomology 2014; 51: 269-277.

9. Ochieng C, et al. Mosquito-borne arbovirus surveillance at selected sites in diverse ecological zones of Kenya; 2007-2012. Virology Journal 2013; 10: 140.

10. Jost $\mathbf{H}$, et al. Isolation and phylogenetic analysis of Batai virus, Germany. American Journal of Tropical Medicine and Hygiene 2011; 84: 241-243.

11. Yanase T, et al. Genetic characterization of Batai virus indicates a genomic reassortment between orthobunyaviruses in nature. Archives of Virology 2006; 151: $2253-2260$

12. Standley K. MAFFT multiple sequence alignment software version 7: improvements in performance and usability. Molecular Biology and Evolution 2013; 30: $772-780$

13. Tamura K, et al. MEGA5: molecular evolutionary genetics analysis using maximum likelihood, evolutionary distance, and 336 maximum parsimony methods. Molecular Biology and Evolution 2011; 28: 2731-2739.

14. Huhtamo E, et al. Isolation and full genomic characterization of Batai virus from mosquitoes, Italy 2009. Journal of General Virology 2013; 94: 1242-1248. 


\section{Supplementary Material}

\section{Supplementary Table S1. Primers used in sequencing of Kenyan Bunyamwera and Ngari virus isolates}

\begin{tabular}{|c|c|c|c|}
\hline Target protein & Primer sequence (5'-3') & $\begin{array}{l}\text { Annealing } \\
\text { position (5'-3') }\end{array}$ & Reference \\
\hline \multicolumn{4}{|l|}{ BUNV N protein } \\
\hline BUNS1 & AGTAGTGTACTCCACACTACAAACT & $1-25$ & Yandoko et al, $2007[1]$ \\
\hline BUNS9 & AGGAATCCACTGAGGCGGTGGAGG & $381-358$ & \\
\hline BUNS4 & CTGGCAACCGGAACAACCCAGTT & $318-340$ & Yandoko et al, $2007[1]$ \\
\hline BUNS5 & GAGACAACTGTCAGTGCAGACTGAA & $711-687$ & \\
\hline BUNS10 & TCAGTCTGCACTGACAGTTGTCTC & $688-711$ & Yandoko et al, $2007[1]$ \\
\hline BUNS2 & AGTAGTGTGCTCCACCTAAAACTTA & $961-937$ & \\
\hline \multicolumn{4}{|l|}{$\begin{array}{l}\text { BUNV } \\
\text { M Polyprotein }\end{array}$} \\
\hline Bunya M14C & CGGAATTCAGTAGTGTACTACC** & $1-14$ & Yanase et al, $2006[2]$ \\
\hline Bunya M619 & GACATATGYTGATTGAAGCAAGCATG* & $586-576$ & \\
\hline BUNM11F & TCAGCACTGGCTGGTTTAAG & $481-500$ & This study \\
\hline BUNM11R & ACCTGCACCGAAGAGTGATG & $1178-1159$ & \\
\hline BunM12F & GAGATAGCAGATGTCCTTCAAGC & $1092-1114$ & This study \\
\hline BunM12R & CAAGCAAGTGCATTCTGTGG & $1725-1706$ & \\
\hline Bun M3F & TTCACTAATAAATGTGGGTTTTG & 1311-1333 & This study \\
\hline Bun M3R & ATGCTGACTGCCTGATAGGG & $2071-2052$ & \\
\hline Bun M4F & TCCAGGCACTGCTTATGTTC & $2003-2022$ & This study \\
\hline Bun M4R & TCTTCCCCTGGTATGTGGTC & $2671-2652$ & \\
\hline Bun M5F & TGTGATTCTGGTCCGCTCTAC & $2625-2645$ & This study \\
\hline Bun M5R & GCATCCCCATCTGCTAGTTC & $3236-3217$ & \\
\hline Bun M6F & CAAAGCATGACGAACATTGC & $3136-3155$ & This study \\
\hline Bun M6R & TCAAGGCTGCAGATTGTGTC & $3919-3900$ & \\
\hline Bunc7MF & CCAGATTGATGCGAAATGTG & $3821-3840$ & This study \\
\hline BAT 3' end R & GAATTCAGTAGTGTGCTACC* & $4458-4445$ & \\
\hline \multicolumn{4}{|l|}{$\begin{array}{l}\text { BUNV } \\
\text { L Protein }\end{array}$} \\
\hline M13 BunL 1C Yanase & TAAAACGACGGCCAGTAGTGTACTCCT ${ }^{*}$ & $1-14$ & Yanase et al, 2006 [2] \\
\hline BunL605R Yunase & RGTGAARTCNCCATGTGC & $614-597$ & \\
\hline Bun2LF & GTTGCTGGACAAGTTTGCTG & $551-570$ & This study \\
\hline Bun2LR & TTGCCAATCTTAACCGCTTC & $1219-1200$ & \\
\hline Bun3LF & AAAATGATTGCCAGGTCAAC & $1152-1171$ & This study \\
\hline Bun3LR & TATGAAAAGCCCAGGTGATG & $1889-1870$ & \\
\hline Bun5LF & AAGAAACAACATGTCAATCTACC & $2421-2443$ & This study \\
\hline Bun5LR & TAGCGGATTTCCTCTTCTGC & $3085-3066$ & \\
\hline Bunya L For JV & AATATAATAGACATAATACATTTAGAGT & $1617-1645$ & This study \\
\hline Bunya L Rev JV & CTCCATTTDGACATRTCTGCA) & $3175-3154$ & \\
\hline BUNL6F & GCTGAACACGGACGAGATG & $3003-3020$ & This study \\
\hline BUNL6R & GGCCCAATGTAAGCACAATC & $3784-3765$ & \\
\hline BUNL7F & GGAGAACCACTATCTGTCTTTGG & $3717-3739$ & This study \\
\hline BUNL7R & ATTGTGCTGGGTTTTGGATG & $4491-4472$ & \\
\hline BUNL8F & GCAGTTCATGCAATCTGTCC & $4412-4431$ & This study \\
\hline BUNL8R & TGTTGCCCTGAATCAATGTG & $5163-5144$ & \\
\hline BUNL9F & TCACAGCTGCAAACACTTCC & $4729-4748$ & This study \\
\hline BUNL9R & TCAATCTGCTTGCCTCTTCC & $5334-5315$ & \\
\hline BUNL10F & AAGCGAGCAAATAATAGCAAATG & $5256-5274$ & This study \\
\hline BUNL10R & TACCTAGGGCCTCTGGATTG & $5866-5847$ & \\
\hline
\end{tabular}




\begin{tabular}{|c|c|c|c|}
\hline BUNL11F & CCGGCTATTTAAGATCAATAAGG & $5575-5597$ & This study \\
\hline BUNL11R & TGCTCATCGCCATTACATTC & $6187-6168$ & \\
\hline BUNL12F & TCTAGCATTGTCCCGTTTTG & $6138-6157$ & This study \\
\hline BUNL12R & TTTTGCCACATAGTGCTTTTG & $6823-6803$ & \\
\hline BUNL12F & TCTAGCATTGTCCCGTTTTG & $6138-6157$ & This study \\
\hline Bun 3'end LR & GTAAAACGACGGCCAGTAGTGTGCTCC** & $6875-6863$ & \\
\hline \multicolumn{4}{|c|}{$\begin{array}{l}\text { NRIV Polyprotein M } \\
\text { segment }\end{array}$} \\
\hline BunyaM14C & CGGAATTCAGTAGTGTACTACC** & $1-14$ & This study \\
\hline TrialR2 & TGACCCGCAATTTGTAAAGG & $845-826$ & \\
\hline BATM13F & CCAAACCGAGAAGTTGAACC & $419-438$ & This study \\
\hline BATM13R & AATCCTTCCAGGACATCAGC & $1099-1080$ & \\
\hline BATM14F & TGCTCATGCTGTGGTCTAGC & $798-817$ & This study \\
\hline BATM14R & ACCTCCACTTTGCCTGTGAG & $1788-1769$ & \\
\hline BATAIM3F & CCTGGGGAAGCATTGTGATTACT & $1704-1726$ & Jost et al, 2011 [3] \\
\hline BATAIM3R & CTAGCCAGCGACTCTTGCCTTCC & $2228-2206$ & \\
\hline BATAIM4F & GTCGCTGGCTAGTGCTACCTCTGG & $2217-2240$ & Jost et al, 2011 [3] \\
\hline BATAIM4R & CTGATTATTGTCGGATTTATTGGGAACCT & $2726-2698$ & \\
\hline BATAIM5F & AAAGGTTCCCAATAAATCCGACAA & $2696-2719$ & Jost et al, 2011 [3] \\
\hline BATAIM5R & CAAATTCTTCACATCCCCAACGACTA & $3220-3195$ & \\
\hline BATAIM6F & AGAATTTGGGTGCCTTGCTGTCA & $3213-3235$ & Jost et al, 2011 [3] \\
\hline BATAIM6R & AGATGTTTGGTCCCCTGTGCTTATTT & 4086-4061 & \\
\hline BAT 3'end F & TGTTCGCAGATAACCATGAAAC & $3688-3709$ & This study \\
\hline BAT 3'end R & GAATTCAGTAGTGTGCTACC* & $4438-4425$ & \\
\hline \multicolumn{4}{|l|}{$\begin{array}{l}\text { NRIV } \\
\text { L segment }\end{array}$} \\
\hline NgariL6F & TTTTGAGAAGGAATAATGAAGG & $5821-5842$ & This study \\
\hline NgariL6R & CCCCTGCTGCAACTAACTTC & $6339-6320$ & \\
\hline
\end{tabular}

* Underlined sequence corresponds to virus segment ends.

\section{References}

1. Yandoko EN, et al. Molecular characterization of African orthobunyaviruses. Journal of General Virology 2007; 88: 1761-1766.

2. Yanase T, et al. Genetic characterization of Batai virus indicates a genomic reassortment between orthobunyaviruses in nature. Archives of Virology. 2006; 151: 2253-2260.

3. Jost $\mathbf{H}$ et al. Isolation and phylogenetic analysis of Batai virus, Germany. American Journal of Tropical Medicine and Hygiene 2011; 84: 241-243. 